\title{
寡聚(4-乙烯基苯基磷酸)/金纳米粒子的磷酸酶可视化检测研究
}

\author{
范艳斌 $a, b$ 陈道勇*, $a, b$ \\ ( ${ }^{a}$ 复旦大学高分子科学系 上海 200433) \\ ( ${ }^{b}$ 聚合物分子工程国家重点实验室 上海 200433)
}

\begin{abstract}
摘要 基于金属纳米粒子的磷酸酶可视化检测技术具有重要的生物医用前景. 然而, 文献中报道的方法大多采用多肽 或核酸等生物分子作为磷酸酶感应基团. 由于这些生物分子在水溶液中不稳定，且可被磷酸酶以外的生物酶类降解， 导致这些方法在实际应用中存在局限性. 首次合成了一种化学结构稳定的水溶性寡聚(4-乙烯基苯基磷酸) [oligo(4-vinyl-phenyl phosphate), OVPP], 并制备得到由 OVPP 稳定的磷酸酶响应型金纳米粒子Au@OVPP, 最后以酸性 磷酸酶为例, 考察了 $\mathrm{Au} @ \mathrm{OVPP}$ 在磷酸酶可视化检测方面的应用. 由于 OVPP 的亲水性, Au@OVPP 可在水溶液中稳定 分散; 当体系中存在磷酸酶时, 由于 OVPP 中的亲水基团(磷酸根)被磷酸酶水解释放, 使得 Au@OVPP 的稳定分子 OVPP 由亲水变为疏水, 进而导致金纳米粒子的聚集及溶液宏观颜色的改变. 通过(肉眼)观察 Au@OVPP 溶液的变色及 程度，建立了一种灵敏、专一和低成本的磷酸酶检测方法. 该方法对于酸性磷酸酶的(肉眼)检测下限约为 $10 \mathrm{U} / \mathrm{mL}$, 且 对多种生物分子具有较好的抗干扰能力.
\end{abstract}

关键词 金纳米粒子; 磷酸衍生物; 磷酸酶; 可视化; 检测

\section{Oligo(4-Vinyl-Phenyl Phosphate)/Gold Nanoconjugate for Visual Detection of Phosphatases}

\author{
Fan, Yanbin ${ }^{a, b}$ Chen, Daoyong*,a,b
}

( ${ }^{a}$ Department of Macromolecular Science, Fudan University, Shanghai 200433)

( ${ }^{b}$ State Key Laboratory of Molecular Engineering of Polymer, Shanghai 200433)

\begin{abstract}
Colorimetric assay taking advantage of the unique surface plasmon resonance properties of metallic nanoparticles has attracted significant attention in bioassays due to its simplicity, low cost, high sensitivity and compatibility. However, most reported colorimetric methods used adenosine triphosphate (ATP), peptides or nucleotides (DNA and RNA) as the phosphatase-sensing species, which are quite unstable or hydrolysable by other enzymes in normal conditions. In this paper, a chemically stable and hydrophilic oligo(4-vinyl-phenyl phosphate) (OVPP) was synthesized. Specifically, the oligomer precursor oligo(dibenzyl-4-vinylphenyl phosphate) was synthesized via RAFT polymerization of a synthetic monomer dibenzyl-4-vinylphenyl phosphate in dioxane, using $S$-ethyl- $S^{\prime}-\left(\alpha, \alpha^{\prime}\right.$-dimethyl- $\alpha^{\prime \prime}$-acetic acid) trithiocarbonate as the chain transfer agent. Deprotection by trimethylsilyl bromide in deuterochloroform containing 2,4,6-collidine at $0{ }^{\circ} \mathrm{C}$ and then hydrolysis in water at $\mathrm{pH} 9.0$ gives the desired OVPP. Then, an OVPP-stabilized and phosphatase-responsive gold nanoparticle was prepared via simple ligand exchange in water and finally tested for visual detection of a model phosphatase acid phosphatase (ACP). Due to the hydrophilicity of OVPP, Au@OVPP is soluble and stable in water. In the presence of ACP, however, the hydrophilic phosphate groups in OVPP were hydrolyzed and released, transforming the hydrophilic OVPP of Au@OVPP into hydrophobic type. This change from hydrophilicity to hydrophobicity triggered the aggregation and color changes of the gold nanoparticles and their sols, respectively. Via this method, a sensitive visual detection of ACP was achieved, with a detection limit of $c a .10 \mathrm{U} / \mathrm{mL}$ after $24 \mathrm{~h}$ incubation at $\mathrm{pH} 5.0$ and $37{ }^{\circ} \mathrm{C}$. Besides, a high selectivity for the phosphatase detection was confirmed as controlled with other bio-species including esterase, proteinase, serum proteins, DNA and so on. By using pyrene as a polarity probe, a significant decrease of the ratio $I_{373} / I_{384}$ was found in Au@OVPP sols after incubation with ACP, indicating the hydrolysis of OVPP and formation of hydrophobic domains in the ACP activated solutions. Collectively, Au@OVPP represents a chemically stable, low cost, highly sensitive and specific phosphatase-visualization agent for phosphatase detection.
\end{abstract}

Keywords gold nanoparticle; phosphate derivate; phosphatase; visualization; detection

*E-mail: chendy@fudan.edu.cn; Tel.: 0086-021-65643989

Received May 4, 2014; published July 8, 2014.

Supporting information for this article is available free of charge via the Internet at http://sioc-journal.cn.

Project supported by the National Natural Science Foundation of China (Nos. 21334001 and 91127030) and the Ministry of Science and Technology of China (No. 2011CB932503).

项目受国家自然科学基金(Nos. 21334001, 91127030)及科技部 973 项目基金(No. 2011CB932503)资助. 


\section{1 引言}

生物学上, 磷酸酶通过特异性地水解释放底物上的 磷酸根来改变底物的亲疏水性, 进而实现对底物分子的 构象及功能的调控 ${ }^{[1]}$. 由于磷酸酶广泛参与细胞内的信 号转导过程, 其含量或活性的异常与多种疾病密切相

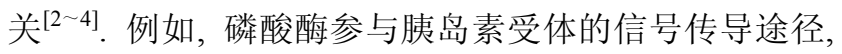
其调控下的底物去磷酸化水平直接影响着上游胰岛素 的刺激响应过程 ${ }^{[3]}$. 因此, 对于磷酸酶含量及其活性的 检测具有重要的临床应用前景 ${ }^{[5 \sim 15]}$.

目前常用的磷酸酶检测技术主要包括抗体法(如免 疫印迹和酶联反应等 $)^{[8]}$ 和底物法 (水解后的底物带有荧 光或紫外吸收 $)^{[9]}$. 例如, 在磷酸酶作用下, 底物硝基苯 酚磷酸酯可被转化成硝基苯酚, 后者在 $405 \mathrm{~nm}$ 处有着 强烈的紫外吸收; 通过检测该处的紫外吸收值, 可实现 对磷酸酶的定量检测 ${ }^{[9]}$. 然而, 这些传统方法存在如下 不足: 一、成本高, 抗体等蛋白试剂通常较为昂贵且容 易失活; 二、无法即时、快速检测, 传统检测方法通常 需要如紫外-可见和荧光光谱仪等大型仪器设备来协助 分析, 且需要专业人员进行操作. 最近, 一种基于金属 (如金和银等)纳米粒子的 “肉眼” 检测技术引起了广泛 的关注 ${ }^{[10 \sim 15]}$, 并有望为上述不足提供解决方案. 该技术 的设计思想为, 利用金属纳米粒子特有的表面等离子共 振性质, 将金属纳米粒子设计成稳定性受磷酸酶调控的 体系, 如磷酸酶能够破坏或重建纳米粒子在溶液中的稳 定性，进而引起金属纳米粒子的聚集或重新分散，同时 伴随着溶液宏观颜色的变化. 通过 “肉眼” 观察这种溶 液的颜色变化, 可方便地对样品中磷酸酶的含量及活性 进行评估. Choi 等 ${ }^{[10]}$ 设计了一种磷酸酶响应的 “电荷反 转型” 金纳米粒子, 即在磷酸酶作用下, 部分金纳米粒 子的表面电荷发生反转(负电荷变为正电荷), 导致该部 分金纳米粒子与溶液中带相反电荷的粒子发生聚集，同 时伴随着溶液颜色的变化. 类似地, $\mathrm{Li}$ 等 ${ }^{[11 ~ 13]}$ 构建了以 三磷酸腺苷(ATP)为基础的磷酸酶检测手段, 即在磷酸 酶作用下, 银纳米粒子的稳定基团 ATP 被转化为二磷 酸腺苷(ADP); 由于 $\mathrm{ADP}$ 不足以稳定银纳米粒子, 从而 导致银纳米粒子聚集以及溶液颜色的变化. 需要指出的 是, 文献中报道的方法大多采用多肽、核酸 ${ }^{[12]}$ 、ATP 或 抗坏血酸 ${ }^{[13]}$ 等生物分子构建磷酸酶检测体系, 由于这 些生物分子的化学结构不稳定 (如 ATP 易分解为 $\mathrm{ADP}^{[16]}$, 而抗坏血酸易被氧化为脱氢抗坏血酸 $)^{[17]}$, 且 多肽和核酸等分子可被其他生物酶类如蛋白酶或核酸 酶等降解，导致这些方法在实际生物样品的测试中存在 局限性.

本文首次合成了一种主链由 $\mathrm{C}-\mathrm{C}$ 键构成的化学结 构稳定的水溶性寡聚(4-乙烯基苯基磷酸) [oligo(4vinyl-phenyl phosphate), OVPP], 并将其修饰到 $10 \mathrm{~nm}$ 金 粒子表面, 制备得到一种由 OVPP 稳定的磷酸酶可视化 检测试剂 $\mathrm{Au} @ O V P P$. 由于 OVPP 的亲水性, Au@OVPP
能够稳定分布在水溶液中; 当溶液中存在磷酸酶时, 由 于 OVPP 中的亲水基团(磷酸根)被磷酸酶水解释放, 使 得 $\mathrm{Au} @ O V P P$ 的稳定分子 OVPP 由亲水变为疏水，进而 导致金纳米粒子的聚集及溶液宏观颜色的改变. 通过肉 眼观察 Au@OVPP 溶液的变色及程度, 建立了一种灵 敏、专一、便捷和低成本的磷酸酶检测方法(图式 1).

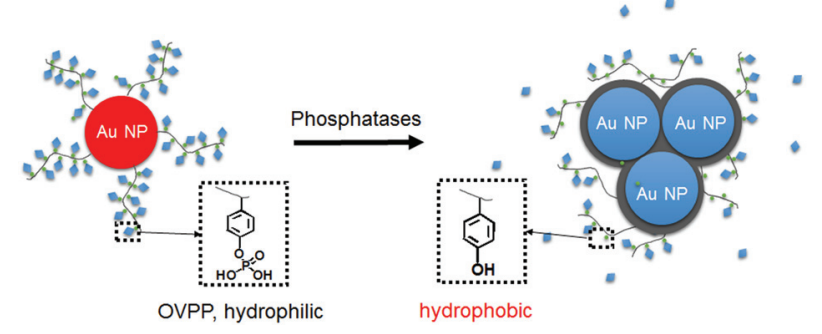

图式 1 基于 $\mathrm{Au} @ O V P P$ 的磷酸酶可视化检测技术的原理示意图 Scheme 1 Schematic illustration of Au@OVPP-based visual detection strategy for phosphatases

\section{2 结果与讨论}

\subsection{OVPP 的合成策略与结构分析}

OVPP 的合成步骤如实验部分 4.1 节中所述. 为了 向 OVPP 中引入与金相互作用的端基(如颈基、二硫键 和三硫酯等), 采用 RAFT 聚合的方法制备端基含有三 硫酯的 OVPP 的前驱体，即臭聚(4-乙烯基苯基磷酸二芐 酯 ${ }^{[18,19]}$. 将该寡聚物进一步硅烷化、水解 ${ }^{[20,21]}$, 即得端 基含有三硫酯的水溶性 OVPP. OVPP 的分子式如图 1A 中所示. 运用核磁 $\left({ }^{1} \mathrm{H},{ }^{31} \mathrm{P}\right.$ NMR) 对 OVPP 的结构进行表 征. 在 OVPP 的 ${ }^{1} \mathrm{H}$ NMR 谱中(图 1A), 位于 $\delta 2.5 \sim 0.6$ 之间的宽峰对应于 RAFT链转移剂上的氢原子的化学位 移; 通过比较该处与位于 $\delta 6.8 \sim 6.0$ 之间峰的积分面积, 得到 OVPP 的聚合度约为 8. 在 OVPP 的 ${ }^{31}$ P NMR 谱中 (图 1B), 只观察到一个位于 $\delta 1.03$ 处的单峰, 根据文献 [20,21], 该单峰对应于 OVPP 中的磷酸基团的化学位 移. 据此, 成功合成出端基含有三硫酯、侧链含有磷酸 单酯的亲水型 OVPP.

\subsection{Au@OVPP 的表征}

在 $\mathrm{pH} 9.0$ 下，借助 OVPP 端基上的三硫酯与金粒子 之间的相互作用，通过配体交换和离心分离的方法制备 OVPP 修饰的金纳米粒子 ${ }^{[22]}$, 即 $\mathrm{Au} @$ OVPP (实验部分 4.1 节). 运用透射电镜(TEM)、动态光散射(dynamic light scattering, DLS)和紫外-可见吸收(UV-Vis)表征 $\mathrm{Au} @ O V P P$. 如图 2A 中所示, Au@OVPP 中的金纳米粒 子为直径在 $10 \pm 0.3 \mathrm{~nm}$ 的球形粒子. DLS 结果显示, $\mathrm{Au} @$ OVPP 的流体力学直径约为 $22 \mathrm{~nm}$, 大于相应的柠 檬酸稳定的金纳米粒子 $(\mathrm{Au} @$ Citrate) 的流体力学直径 (约 $16 \mathrm{~nm}$ ), 表明 OVPP 被成功地修饰到金纳米粒子表 面(图 2B). UV-Vis 结果显示, Au@OVPP 的最大吸收峰 位于 $524 \mathrm{~nm}$, 而相应的 $\mathrm{Au} @$ Citrate 的最大吸收峰位于 

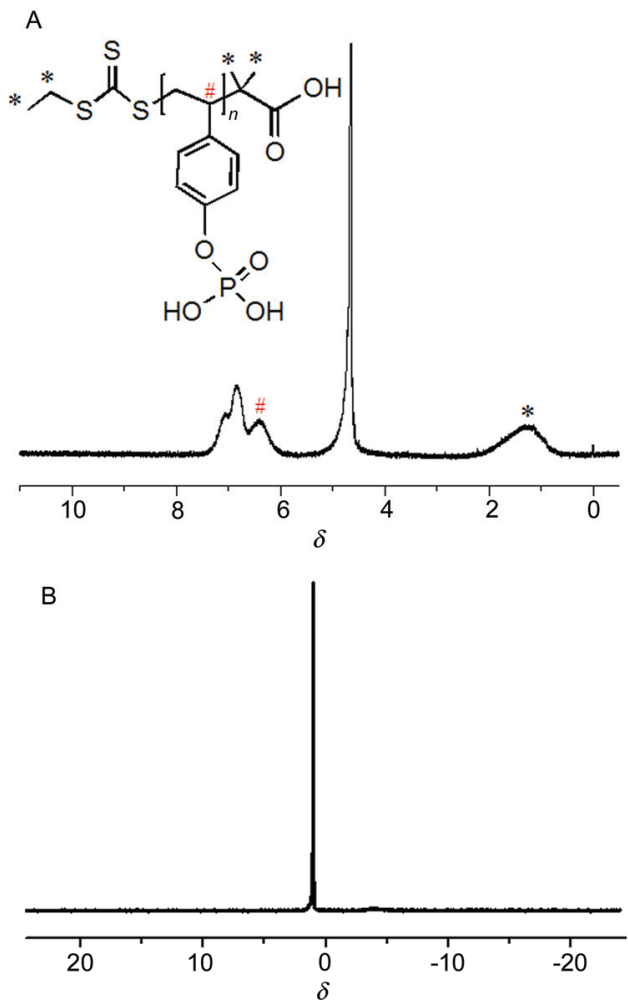

图 1 室温下, OVPP 在氝代水中的 $500 \mathrm{MHz}{ }^{1} \mathrm{H}(\mathrm{A})$ 和 ${ }^{31} \mathrm{P}(\mathrm{B}) \mathrm{NMR}$ 谱 图

Figure $1500 \mathrm{MHz}{ }^{1} \mathrm{H}(\mathrm{A})$ and ${ }^{31} \mathrm{P}(\mathrm{B}) \mathrm{NMR}$ spectra of OVPP in $\mathrm{D}_{2} \mathrm{O}$ at room temperature

$520 \mathrm{~nm}$ (图 S1, supporting information).

\section{3 磷酸酶的可视化检测}

Hawker 等 ${ }^{[20]}$ 通过 ATRP 方法合成了具有磷酸酶响 应性的两嵌段共聚物 PEG-block-OVPP, 发现酸性磷酸 酶(acid phosphatase, ACP) 能够有效作用于嵌段共聚物 中的 OVPP 嵌段, 水解并释放其中的磷酸根基团, 导致 OVPP 嵌段从亲水变为疏水, 进而诱导嵌段共聚物在水 中自组装形成纳米组装体. 本工作中, 我们制备了由 OVPP 稳定的金纳米粒子 (Au@OVPP), 并考察 $\mathrm{Au} @ O V P P$ 在磷酸酶可视化检测方面的应用. 以 ACP 作 为模型磷酸酶, 首先考察含有 $\mathrm{ACP}$ 的 $\mathrm{Au} @ \mathrm{OVPP}$ 溶液 的变色行为. 如图 $3 \mathrm{~A}$ 中所示, 将不同浓度的 $\mathrm{ACP}$ 与 5.0 $\mathrm{nmol} / \mathrm{L}$ 的 $\mathrm{Au} @ \mathrm{OVPP}$ 在 $37{ }^{\circ} \mathrm{C}$ 下培养 $24 \mathrm{~h}$ 后, $\mathrm{Au} @ \mathrm{OVPP}$ 溶液出现了不同程度的变色现象, 且随着 $\mathrm{ACP}$ 含量的增加, 变色现象更加明显. 作为对照, 将与 变色样品中等量的经过变性处理 $\left(60{ }^{\circ} \mathrm{C}, 1.0 \mathrm{~h}\right)$ 的 $\mathrm{ACP}$ 加入 $5.0 \mathrm{nmol} / \mathrm{L} \mathrm{Au} @ \mathrm{OVPP}$ 溶液中, 并在与上述实验相 同条件下培养 $24 \mathrm{~h}$ 后, 未观察到明显的变色现象, 表明 上述 $\mathrm{Au} @ \mathrm{OVPP}$ 溶液的变色由 $\mathrm{ACP}$ 的酶解作用导致. 值得注意的是，当溶液中 ACP 的含量大于或等于 10 $\mathrm{U} / \mathrm{mL}$ 时, 肉眼能够有效观察到溶液的变色现象(图 3A). 因此, 该方法对于 $\mathrm{ACP}$ 的 (肉眼)检测下限约为 10 $\mathrm{U} / \mathrm{mL}$ (约 $0.02 \mathrm{mg} / \mathrm{mL}$ ).

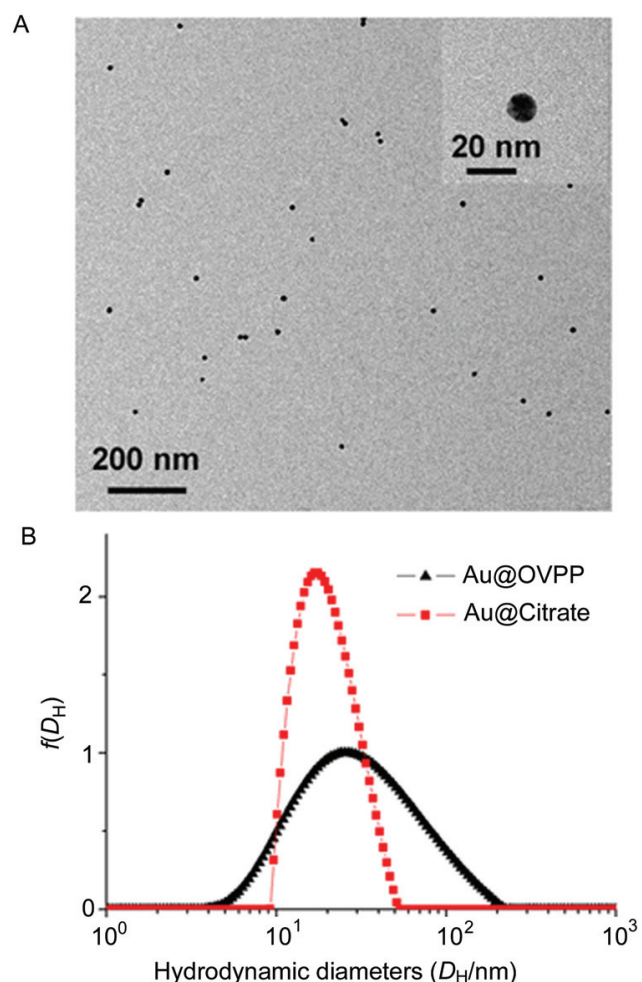

图 2 室温下, $1.0 \mathrm{nmol} / \mathrm{L}$ 的 $\mathrm{Au} @ \mathrm{OVPP}$ 在水中的 TEM 照片(A), 以及 $1.0 \mathrm{nmol} / \mathrm{L} \mathrm{Au} @ O V P P$ 或 $1.0 \mathrm{nmol} / \mathrm{L} \mathrm{Au} @$ Citrate 在水中的流体力学直 径分布曲线(B, DLS 测定)

Figure 2 A typical TEM image of Au@OVPP $(1.0 \mathrm{nmol} / \mathrm{L})$ in water (A) and the hydrodynamic diameter distribution profiles of Au@OVPP $(1.0 \mathrm{nmol} / \mathrm{L})$ or $\mathrm{Au} @$ Citrate $(1.0 \mathrm{nmol} / \mathrm{L})$ in water as revealed by DLS (B) at room temperature
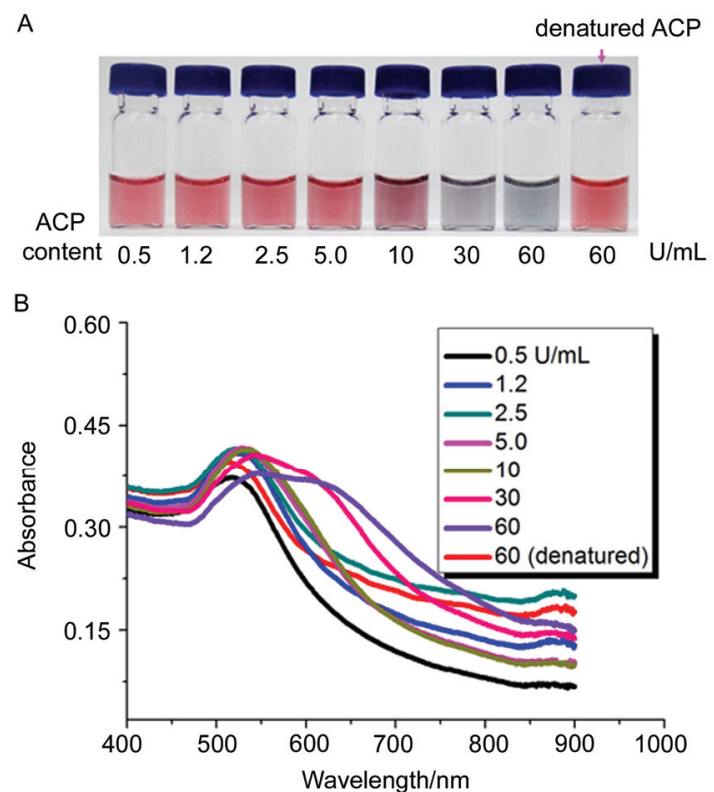

图 $337{ }^{\circ} \mathrm{C}$ 下, 将 $0.7 \mathrm{~mL}$ 含有 $5.0 \mathrm{nmol} / \mathrm{L} \mathrm{Au} @ \mathrm{OVPP}$ 的柠檬酸钠缓 冲溶液 $(5.0 \mathrm{mmol} / \mathrm{L}, \mathrm{pH} 5.0$ )与不同量的 $\mathrm{ACP}$ 共培养 $24 \mathrm{~h}$ 后的照片(A), 以及相应样品的紫外-可见吸收光谱(B)

Figure 3 A photograph of vials containing $0.7 \mathrm{~mL}$ citrate buffer $(5.0$ $\mathrm{mmol} / \mathrm{L}, \mathrm{pH} 5.0$ ) suspended with $5.0 \mathrm{nmol} / \mathrm{L} \mathrm{Au} @ O V P P$ in each vial after incubation with different contents of ACP for $24 \mathrm{~h}$ at $37{ }^{\circ} \mathrm{C}$ (A), and the corresponding UV-Vis spectra at room temperature (B) 
测定上述样品的紫外-可见吸收光谱(UV-Vis, 图 $3 \mathrm{~B})$. 结果显示, ACP 的加入使得 $\mathrm{Au} @ \mathrm{OVPP}$ 的最大吸收 波段发生显著的红移, 且波谱呈现增宽现象, 表明溶液 中出现了较大尺寸的金纳米粒子的聚集体 ${ }^{[23]}$. 运用 DLS 和 TEM 研究磷酸酶作用后溶液中金纳米粒子的流 体力学直径和形貌(图 S2, supporting information). 结果 表明, 磷酸酶作用后, 溶液中金纳米粒子的流体力学直 径显著增加, 且通过 TEM 观测到了较大尺寸的金纳米 粒子的聚集体，证明了溶液中金纳米粒子的聚集现象. 如前所述, ACP 能够有效作用于 Au@OVPP 中的 OVPP 部分, 水解并释放其中的亲水基团磷酸根, 使得 $\mathrm{Au} @ \mathrm{OVPP}$ 的稳定基团由亲水变为疏水, 进而导致 $\mathrm{Au} @ \mathrm{OVPP}$ 的聚集和溶液颜色的改变. 进一步地, 选用 萠探针表征 ACP 作用前后的 Au@OVPP 溶液中亲疏水 环境的变化(图 S3, supporting information). 结果显示, $\mathrm{ACP}$ 作用后, $\mathrm{Au} @ \mathrm{OVPP}$ 溶液中的 $I_{373} / I_{384}$ 比值显著降低, 表明溶液中的疏水区域增多, 进一步证实上述金纳米粒 子的聚集由 ACP 的酶解作用导致.

由上可知, 随着 $\mathrm{Au} @ \mathrm{OVPP}$ 溶液变色(聚集)程度的 增加, 其最大吸收波段由原来的 $524 \mathrm{~nm}$ 红移至约 600 $\mathrm{nm}$ 左右, 因此, 524 和 $600 \mathrm{~nm}$ 处吸光度的比值 $\left(A_{524} / A_{600}\right)$ 可用于表征溶液中金纳米粒子的变色 (聚集)程度 ${ }^{[10 \sim 15]}$. 相应地，本文计算了上述样品的 UV-Vis 吸收光谱中 524 和 $600 \mathrm{~nm}$ 处吸光度的比值 $\left(A_{524} / A_{600}\right)$, 并将该比值与 $\mathrm{ACP}$ 的浓度进行作图, 得到图 4 中所示曲线. 可见, $A_{524} / A_{600}$ 的值与 $\mathrm{ACP}$ 的浓度成负相关, 即随着 $\mathrm{ACP}$ 浓度 的增加, $A_{524} / A_{600}$ 的数值逐渐减小, 对应于溶液的变色程 度增加. 由此, 得到了 $\mathrm{Au} @ \mathrm{OVPP}$ 溶液的变色程度与溶 液中磷酸酶含量之间的半定量关系, 即变色程度越大 $\left(A_{524} / A_{600}\right.$ 值越小), 磷酸酶的含量(活性)越高.

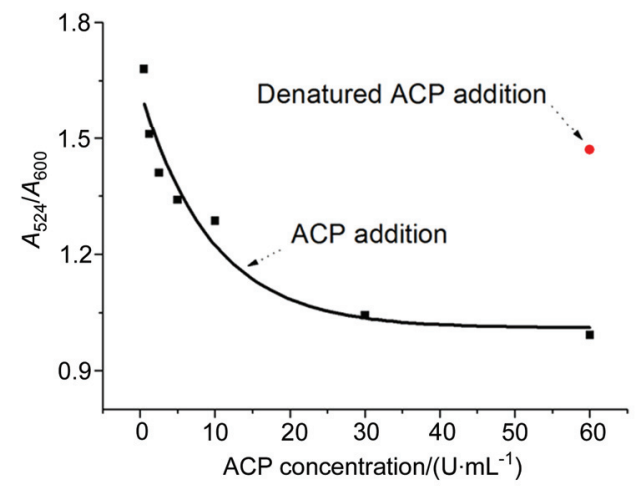

图 $437^{\circ} \mathrm{C}$ 下, 不同浓度的 $\mathrm{ACP}$ 与 $5.0 \mathrm{nmol} / \mathrm{L} \mathrm{Au} @ O V P P$ 共培养 $24 \mathrm{~h}$ 后的 $A_{524} / A_{600}$ 值与 $\mathrm{ACP}$ 浓度的依赖性作图

Figure $4 A_{524} / A_{600}$ value versus ACP concentration plotting after the incubation of $5.0 \mathrm{nmol} / \mathrm{L} \mathrm{Au} @ \mathrm{OVPP}$ with various concentrations of ACP for $24 \mathrm{~h}$ at $37^{\circ} \mathrm{C}$

\section{4 专一性}

为了检验 $\mathrm{Au} @$ OVPP 的磷酸酶可视化检测的专一 性, 在与上述实验相同的条件下, 将不同的生物分子与
Au@OVPP 共培养 24 h. 如图 5 中所示, 将 Au@OVPP 与 $\mathrm{ACP}$ 共培养时, 溶液发生显著变色(图 5A), 且溶液中 $A_{524} / A_{600}$ 值显著降低(图 5B); 而当 $\mathrm{Au} @ O V P P$ 与相同质 量分数的 ATP、牛血清白蛋白(BSA)、胰蛋白酶(Trypsin, Trp)、麦芽脂酶(wheat germ lipase, WGL)或 DNA 共培养 时, 溶液无明显的变色现象发生 $\left(A_{524} / A_{600}\right.$ 值无明显下 降), 表明 $\mathrm{Au} @$ OVPP 的磷酸酶可视化检测具有显著的 专一性.

A

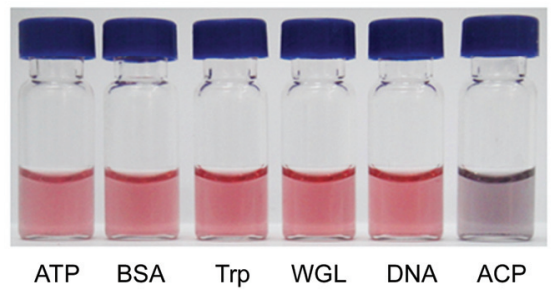

B

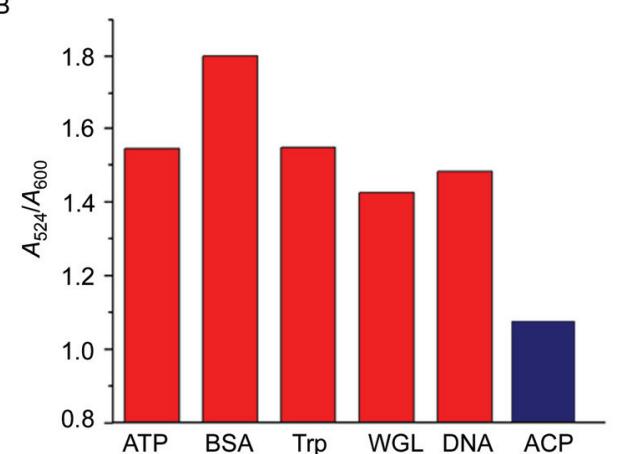

图 $537{ }^{\circ} \mathrm{C}$ 下, 将 $0.7 \mathrm{~mL}$ 含有 $5.0 \mathrm{nmol} / \mathrm{L} \mathrm{Au} @ \mathrm{OVPP}$ 的柠檬酸钠缓 冲溶液 $(5.0 \mathrm{mmol} / \mathrm{L}, \mathrm{pH} 5.0)$ 与不同生物分子 $(0.02 \mathrm{mg} / \mathrm{mL})$ 共培养 $24 \mathrm{~h}$ 后的照片(A), 以及相应样品的 $A_{524} / A_{600}$ 数值(B)

Figure 5 A photograph of vials containing $0.7 \mathrm{~mL}$ citrate buffer $(5.0$ $\mathrm{mmol} / \mathrm{L}, \mathrm{pH} 5.0$ ) suspended with $5.0 \mathrm{nmol} / \mathrm{L}$ Au@OVPP in each vial after incubation with different bio-species $(0.02 \mathrm{mg} / \mathrm{mL})$ for $24 \mathrm{~h}$ at $37{ }^{\circ} \mathrm{C}$ (A), and the corresponding $A_{522} / A_{600}$ values (B)

\section{3 结论}

合成了一种化学结构稳定的水溶性寡聚(4-乙烯基 苯基磷酸)(OVPP), 将 OVPP 与金纳米粒子复合制备得 到一种由 OVPP 稳定的磷酸酶可视化检测试剂 Au@OVPP. 在磷酸酶作用下, Au@OVPP 的水溶液颜色 发生改变, 且磷酸酶含量(活性)越高, 溶液的变色程度 越大. 借助于这种溶液的变色程度与磷酸酶含量之间的 半定量关系，建立了一种灵敏、专一、便捷和低成本的 磷酸酶可视化检测方法. 相比于同类试剂而言, Au@OVPP 具有化学结构稳定和抗生物分子干扰能力 强等特点.

\section{4 实验部分}

\subsection{Au@OVPP 的制备}

参照文献分别合成单体 4-乙烯基苯基磷酸二芐 酯 ${ }^{[18]}$ 和 RAFT 聚合反应链转移剂 $S$-ethyl- $S^{\prime}-\left(\alpha, \alpha^{\prime}-\right.$ di- 
methyl- $\alpha$ "-acetic acid)trithiocarbonate (RAFT CTA) ${ }^{[19]}$. 准确称取 $6.6 \mathrm{mg}$ RAFT CTA $(29.25 \mu \mathrm{mol}), 1.0 \mathrm{mg}$ 偶氮 二异丁腈(AIBN, 重结晶, $6 \mu \mathrm{mol}$ )和 $1.1119 \mathrm{~g}$ 单体(2.925 $\mathrm{mmol}$ )并溶于 $2.0 \mathrm{~mL}$ 二氧六环中, 小心转移溶液至配有 磁子的 $20 \mathrm{~mL}$ 安培管中, 冻融脱气五次除氧. 火焰灯下 封管后, 将溶液温度升至 $70{ }^{\circ} \mathrm{C}$, 并于氩气氛下反应 $8 \mathrm{~h}$. 反应结束后, 加入 $2 \mathrm{~mL}$ 氯仿稀释反应液, 并于 $20 \mathrm{~mL}$ 冰的无水乙醚中沉淀. 过滤沉淀后, 用约 $2 \mathrm{~mL}$ 氯仿重 新溶解产物, 再于 $20 \mathrm{~mL}$ 冰的无水乙醚中沉淀, 重复此 操作三次. 将所得产物于室温下真空干燥 $48 \mathrm{~h}$, 即得寡 聚(4-乙烯基苯基磷酸二芐酯). 参照文献报道方法 ${ }^{[20,21]}$, 将该寡聚物在 2,4,6-可力丁和三甲基溴硅烷作用下转化 为易水解的硅烷酯, 最后于 $\mathrm{pH} 9.0$ 中水解该硅烷酯, 得 终产物 OVPP.

参照文献制备直径在 $10 \sim 12 \mathrm{~nm}$ 的金纳米粒子 ${ }^{[22]}$. 取约 $20 \mathrm{~mL}$ 制备得到的柠檬酸稳定的金纳米粒子溶液, 离心浓缩至 $4 \mathrm{~mL}\left(13500 \mathrm{r} / \mathrm{min}, 20 \mathrm{~min}, 15{ }^{\circ} \mathrm{C}\right.$; 约 100 $\mathrm{nmol} / \mathrm{L}$ ), 再用 $0.5 \mathrm{~mol} / \mathrm{L} \mathrm{Na}_{2} \mathrm{CO}_{3}$ 的水溶液调节溶液 $\mathrm{pH}$ 至 9.0. 剧烈搅拌下, 逐渐加入 $2 \mathrm{~mL}$ 含有 $12.6 \mathrm{mg} \mathrm{OVPP}$ 的去离子水, 室温下搅拌过夜. 离心分离金纳米粒子后, 加入 $4 \mathrm{~mL}$ 去离子水洗涤并再次离心. 重复此操作三次, 并将最后得到的金纳米粒子重悬于 $4 \mathrm{~mL}$ 去离子水中, 即得 $100 \mathrm{nmol} / \mathrm{L} \mathrm{Au} @$ OVPP 的穴存液.

\subsection{Au@OVPP 与生物分子的相互作用}

用去离子水将 $100 \mathrm{nmol} / \mathrm{L} \mathrm{Au@OVPP}$ 的它存液稀 释至 $10 \mathrm{nmol} / \mathrm{L}$, 再与等体积的柠檬酸钠缓冲液 (10 $\mathrm{mmol} / \mathrm{L}, \mathrm{pH} 5.0$ )混合, 得 $5.0 \mathrm{nmol} / \mathrm{L} \mathrm{Au} @ \mathrm{OVPP}$ 的柠檬 酸钠缓冲溶液 ( $5.0 \mathrm{mmol} / \mathrm{L}, \mathrm{pH} 5.0)$. 分别移取 $0.7 \mathrm{~mL}$ 上 述溶液至 7 个容积为 $2.0 \mathrm{~mL}$ 小瓶中, 并依次加入 0.5 , $1.2,2.5,5.0,10,30$ 及 $60 \mathrm{U} / \mathrm{mL}$ 的 $\mathrm{ACP}$ 至上述小瓶中, 最 后将样品于 $37{ }^{\circ} \mathrm{C}$ 下振荡培养 $(120 \mathrm{r} / \mathrm{min}) 24 \mathrm{~h}$. $\mathrm{Au} @$ OVPP 与其他生物分子 $(0.02 \mathrm{mg} / \mathrm{mL})$ 共培养的操作 步骤同上.

向上述培养液中加入等体积的柠檬酸钠缓冲溶液
(5.0 mmol/L, pH 5.0), 并于常温下测定其 400 900 nm 处的 UV-Vis 吸收曲线(型号 UV2500).

\section{References}

[1] Cohen, P. Nat. Cell Biol. 2002, 4, E127.

[2] Mijakovic, I.; Poncet, S.; Galinier, A.; Monedero, V.; Fieulaine, S.; Janin, J.; Nessler, S.; Marquez, J. A.; Scheffzek, K.; Hasenbein, S.; Hengstenberg, W.; Deutscher, J. Proc. Natl. Acad. Sci. USA 2002, 99, 13442.

[3] Goldstein, B. J.; Bittner-Kowalczyk, A.; White, M. F.; Harbeck, M. J. Biol. Chem. 2000, 275, 4283.

[4] Husberg, C.; Agnetti, G.; Holewinski, R. J.; Christensen, G.; Van Eyk, J. E. Proteomics 2012, 12, 973.

[5] Li, P.; Lu, L. Chin. J. Inorg. Chem. 2013, 29, 1830. (李鹏宇, 卢丽 萍, 无机化学学报, 2013, 29, 1830.)

[6] Zhang, X.; Huang, Y.; Fan, C.; Huang, W. Chem. J. Chin. Univ. 2011，32，2548. (张小平，黄艳琴，樊春海，黄维，高等学校化学 学报, 2011, 32, 2548.)

[7] Gao, X.; Lu, L.; Zhu, M.; Yuan, C.; Ma, J.; Fu, X. Acta Chim. Sinica 2009, 67, 929. (高晓丽, 卢丽萍, 朱苗力, 袁彩霞, 马俊锋, 付学 奇, 化学学报, 2009, 67, 929.)

[8] Bross, P.; Naundrup, S.; Hansen, J.; Nielsen, M. N.; Christensen, J. H.; Kruhoffer, M.; Palmfeldt, J.; Corydon, T. J.; Gregersen, N.; Ang, D.; Georgopoulos, C.; Nielsen, K. L. J. Biol. Chem. 2008, 283, 15694.

[9] Iqbal, J. Anal. Biochem. 2011, 414, 226.

[10] Choi, Y.; Ho, N. H.; Tung, C. H. Angew. Chem., Int. Ed. 2007, 46, 707.

[11] Wei, H.; Chen, C.; Han, B.; Wang, E. Anal. Chem. 2008, 80, 7051.

[12] Zhao, W.; Chiuman, W.; Lam, J. C.; Brook, M. A.; Li, Y. Chem. Commun. 2007, 36, 3729.

[13] Li, C. M.; Zhen, S. J.; Wang, J.; Li, Y. F.; Huang, C. Z. Biosens. Bioelectron. 2013, 43, 366.

[14] Jiao, H.; Chen, J.; Li, W.; Wang, F.; Zhou, H.; Li, Y.; Yu, C. ACS Appl. Mater. Interfaces 2014, 6, 1979.

[15] Hayat, A.; Gonca, B.; Andreescu, S. Biosens. Bioelectron. 2014, 56, 334.

[16] Chance, B.; Lees, H.; Postgate, J. G. Nature 1972, 238, 330.

[17] Widengren, J.; Chmyrov, A.; Eggeling, C.; Löfdahl, P.-Å.; Seidel, C. A. J. Phys. Chem. A 2007, 111, 429.

[18] Andrus, M. B.; Liu, J. Tetrahedron Lett. 2006, 47, 5811.

[19] Convertine, A. J.; Lokitz, B. S.; Vasileva, Y.; Myrick, L. J.; Scales, C. W.; Lowe, A. B.; McCormick, C. L. Macromolecules 2006, 39, 1724.

[20] Amir, R. J.; Zhong, S.; Pochan, D. J.; Hawker, C. J. J. Am. Chem. Soc. 2009, 131, 13949.

[21] Fan, Y.; Li, F.; Chen, D. Biomaterials 2014, 35, 7870.

[22] Jana, N. R.; Gearheart, L.; Murphy, C. J. Langmuir 2001, 17, 6782.

[23] Nam, J.; Won, N.; Jin, H.; Chung, H.; Kim, S. J. Am. Chem. Soc. 2009, 131, 13639. 